\title{
Circulation of DENV-3 Genotype 3 during 2017 to 2018 in Delhi: A Single-Center Hospital-Based Study
}

\author{
Abhishek Padhi ${ }^{1}$ Ekta Gupta ${ }^{1}$ Gaurav Singh ${ }^{1}$ Shama Parveen ${ }^{2}$ Arshi Islam ${ }^{2}$ Bansidhar Tarai ${ }^{3}$
}

${ }^{1}$ Department of Clinical Virology, Institute of Liver and Biliary Sciences, New Delhi, Delhi, India

${ }^{2}$ Centre for Interdisciplinary Research in Basic Sciences, Jamia Millia Islamia, New Delhi, Delhi, India

${ }^{3}$ Department of Microbiology and Infection Control, Max

Superspeciality Hospital, New Delhi, Delhi, India

\begin{abstract}
Address for correspondence Ekta Gupta, MD, Department of Clinical Virology, Institute of Liver and Biliary Sciences, New Delhi - 110070, Delhi, India (e-mail: ektagaurisha@gmail.com).
\end{abstract}

J Lab Physicians 2022;14:21-26.

\begin{abstract}
Keywords

- dengue

- DENV-3

- genotype 3

- surveillance

Introduction Delhi is hyperendemic for dengue virus (DENV) where all the four DENV have previously been reported. A constant vigilance of circulating DENV serotypes is important in surveillance, since the introduction of a new variant to areas affected by preexisting serotypes constitutes a risk factor for dengue hemorrhagic fever and dengue shock syndrome.

Objectives This retrospective study was performed with an objective to determine the circulating serotype and genotype of DENV in acute phase blood samples of patients who have reported to a tertiary liver care hospital in New Delhi during the last 2 years (2017-2018).

Methods The data of clinician-initiated testing for dengue nonstructural protein 1 (NS1) antigen (Ag) was searched in the institutional hospital information system. The serum sample of dengue NS1 Ag-positive cases confirmed by enzyme-linked immunosorbent assay (ELISA; PANBIO, Gyeonggi-do, ROK) and a fever duration of less than 5 days were retrieved from the laboratory archive. The DENV serotyping on these sample was performed by reverse transcriptase polymerase chain reaction (RT-PCR). Sequencing and phylogenetic analysis was done for the capsid premembrane (CprM) region to determine the genotype.

Results A total of 440 acute-phase samples were received. Twenty one $(4.77 \%)$ were positive for dengue NS1 Ag with a mean age of 35.1 years and male-to-female ratio of 1.1:1. Eight cases (38.09\%) were positive by dengue RT-PCR and all belonged to DENV-3 serotypes. Phylogenetic tree analysis revealed DENV-3 clustered to genotype III with $100 \%$ homology with 2008 Indian subcontinent strain.

Conclusion This study revealed circulation of DENV-3, genotype III in Delhi from 2017 to 2018 , similar to the 2008 viral type. Virological surveillance is an important exercise to be done for viral infections with public threat and outbreak potential.
\end{abstract}

published online September 14, 2021
DOI https://doi.org/

$10.1055 / \mathrm{s}-0041-1734017$. ISSN 0974-2727.
(C) 2021. The Indian Association of Laboratory Physicians. All rights reserved.

This is an open access article published by Thieme under the terms of the Creative Commons Attribution-NonDerivative-NonCommercial-License, permitting copying and reproduction so long as the original work is given appropriate credit. Contents may not be used for commercial purposes, or adapted, remixed, transformed or built upon. (https://creativecommons.org/ licenses/by-nc-nd/4.0/)

Thieme Medical and Scientific Publishers Pvt. Ltd., A-12, 2nd Floor, Sector 2, Noida-201301 UP, India 


\section{Introduction}

Dengue is a vector borne acute arboviral infection widely prevalent in the tropics and subtropics. According to the World Health Organization (WHO) estimates, 3.6 billion people reside in dengue endemic areas and $70 \%$ of the actual burden are in Asia. ${ }^{1,2}$

Symptoms of dengue infection is wide ranging from subclinical disease to severe flu like symptoms, and some people develop severe dengue associated with number of complications such as severe bleeding, organ impairment, and/or plasma leakage. Over the past two decades, the burden of dengue cases has increased almost 15 folds. ${ }^{1}$

Dengue infection is caused by a virus belonging to the family flaviviridae, genus Flavivirus, and consists of a single stranded positive sense RNA approximately $11 \mathrm{~kb}$ as its genome encodes for three structural (capsid [C], premembrane $[\mathrm{prM}]$, and envelope $[\mathrm{E}]$ ) and seven nonstructural proteins $^{3}$. Dengue virus (DENV) can be classified into four antigenically distinct serotypes (DENV-1, DENV-2, DENV-3, and DENV-4) based on sequence diversity and each serotype is further grouped into various genotypes. ${ }^{4}$

A life-long immunity is attained after infection with a particular serotype and a cross-protective immunity against other serotypes for a short period of time. ${ }^{5}$ Secondary infection by a different serotype is associated with severe dengue probably due to antibody-dependent enhancement (ADE) resulting in high viremia. ${ }^{6,7}$

Delhi is hyperendemic for DENV with cocirculation of all the four dengue serotypes. ${ }^{8}$ Delhi has witnessed several dengue epidemics in the past two decades due to rapid boom in urbanization, monsoon-influenced subtropical climate, water storage practices, and use of water coolers. These factors provide the mosquito vector Aedes aegypti a favorable environment to thrive and transmit DENV among the human population which, at times, gives rise to major outbreaks depending on the circulating serotype and genotype of DENV. ${ }^{9,10}$

In the absence of an effective vaccine, virological surveillance and development of effective, locally adapted control programs are the two most important arms in the prevention of dengue infection. Hence, there is a need of continuous monitoring of the circulating DENV type in a given population to develop such locally adapted control programs. Furthermore, most studies focus on the circulating serotypes during epidemics and the studies on circulating strains during the interepidemic periods are neglected and there can be significant variations for which public health systems are unprepared. ${ }^{11}$

We performed the present study targeting the CprM gene junction of the virus to elucidate the molecular epidemiology of the circulating strain of DENV.

\section{Materials and Methods}

\section{Patient Selection and Specimen Collection}

This was a retrospective study performed from January 2017 to December 2018 in a tertiary care liver hospital in New Delhi, India. All consecutive patients with signs and symptoms (according to WHO guidelines) suggestive of DENV infections visiting the outpatient department (OPD) or admitted in inpatient department (IPD) were tested for DENV infection. ${ }^{12}$ Plasma was separated from blood samples and stored in the virology repository at $-80^{\circ} \mathrm{C}$ till further testing.

\section{Dengue Virus Diagnosis}

Diagnosis of DENV was done with dengue NS1 antigen (Ag; PANBIO, Gyeonggi-do, ROK) if the sample was collected within the first 5 days of onset of clinical symptoms, and with dengue immunoglobulin (Ig)-M capture ELISA (PANBIO, Gyeonggi-do, ROK) if the sample was collected after 5 days of onset of clinical symptoms.

\section{RNA Extraction and cDNA Synthesis}

All the DENV NS1 Ag-positive cases were further processed for RNA extraction using QIAamp Viral Mini kit (Qiagen, $\mathrm{GmBH}$, Germany) according to the manufactures' instructions. The extracted viral RNA was reverse transcribed to cDNA using Quantitect reverse transcriptase kit (QIAGEN, GmBH Germany) according to manufacturer's instructions.

\section{Detection of Dengue Virus by Reverse Transcriptase Polymerase Chain Reaction}

The cDNA was then amplified in the external round of reverse transcriptase polymerase chain reaction (RT-PCR) using DENV consensus primers (D1: 5' TCAATATGCTGAAACGCGCGAGAAACC G 3'; D2: 5' TTGCACCAACAGTCAATGTCT TCA GGT TC $3^{\prime}$ ) in $25-\mu \mathrm{L}$ reaction volume. The DNA product obtained was of $511 \mathrm{bp}$.

Subsequent amplification of cDNA was performed with the DENV consensus forward primer (D1) and four dengue serotype-specific reverse primers (TS 1: $5^{\prime}$ CGTCTCAGTGATCCGGGGA 3'; TS 2: 5' CGCCACAAGGGCCATGAACAG 3'; TS 3: 5' TAACATCAT- CATGAGACAGAGC 3'; TS 4: 5' CTCTGTTGTCTTAAACAAGAGA $3^{\prime}$ ) as described by Lanciotti et al, ${ }^{13}$ but all the four serotype-specific primers were added in a single-reaction mixture. A 1:20 dilution of the external PCR product was used in the nested PCR reaction. DENV serotypes were identified by the size of the resulting DNA bands (DENV-1: $482 \mathrm{bp}$, DENV-2: $119 \mathrm{bp}$, DENV-3: $290 \mathrm{bp}$, and DENV-4: $392 \mathrm{bp}$ ). Amplicons were resolved on 2\% agarose gel and visualized with ethidium bromide in ultraviolet (UV) light by using a gel documentation system (Wealtec, United States).

\section{DNA Sequencing}

PCR amplicons were gel-purified using QIAquick gel extraction kit (QIAGEN, GmBH Germany) as per manufacturer's instructions. Purified amplicons were subjected to Sanger's sequencing using ABI 3730 DNA analyzer (Thermofisher Scientific, Waltham, Massachusetts, United States).

\section{Phylogenetic Analysis}

BLAST tool was used to confirm the identity of the obtained sequences (http://blast.ncbi.nlm.nih.gov/Blast.cgi, NCBI). Forward and reverse sequence alignments were done manually and edited to resolve the nucleotide ambiguities and 
consensus sequences were obtained using GeneDoc v.2.7 (http://genedoc.software.informer.com/2.7/) and BioEdit v.7.2 (http://bioedit.software.informer.com/7.2/). All the new sequences of DENV-3 genome were submitted to Genbank.

Maximum likelihood method using MEGA 6 v.6.06 software was used for constructing the phylogenetic tree for the DENV-3 sequences. Tamura-Nei model of nucleotide substitution was used to calculate genetic distances. A total of 1,000 bootstrap replicates were used to assess the robustness of the tree.

\section{Ethics}

The study had the approval of the Institutional Ethical Committee and was conducted following the Declaration of Helsinki. Patient's consent was waived-off, as it was done on the deidentified and anonymized left over clinical samples.

\section{Results}

A total of 440 acute-phase samples were received. Twenty one (4.77\%) were positive for dengue NS1 Ag with a mean age of 35.1 years and male-to-female ratio of $1.1: 1$. Eight cases (38.09\%) were positive by dengue RT-PCR and all belonged to DENV-3 serotypes. No case of concomitant infection with more than one serotype was observed. In cases where NS1 Ag was positive but RT-PCR was negative, duration of fever was 5 days, as by this time viremia declines and only NS1 Ag persists till IgM antibodies are formed. The window for viral RNA detection is limited to 4 to 5 days after the initial infection and NS1 Ag stays in the circulation for a period of 9 to 10 days. ${ }^{14,15}$ Therefore, at day 5, postinfection RT-PCR might miss few samples and can be picked by Ag test.

\section{DNA Sequence Analysis}

The CprM region of the DENV-3 genome was sequenced for eight positive samples. The samples were sequenced in both forward and reverse direction. All the obtained DENV-3 sequences were confirmed by BLAST. These sequences are submitted in Genbank database and accession number of the same are awaited.

\section{Phylogenetic Analysis}

Phylogenetic analysis was done for DENV-3 serotype for the present study. The prototype strain used was H87 strain of genotype V of DENV-3 (GenBank Accession number M93130), corresponding to 131 to $370 \mathrm{bp}$ of the CprM region of full genome of the prototype strain. Eighty sequences ( 8 studied and 72 other sequences of different genotypes of DENV-3 (GenBank) were aligned to construct maximum likelihood tree. Phylogenetic analysis clustered all the studied sequences with the genotype III (Indian subcontinent; - Fig. 1).

\section{Discussion}

Delhi, the capital of India, is known to be hyperendemic for DENV infection due to the circulation of all the four serotypes in the population. ${ }^{10}$ In the past two decades, Delhi has witnessed several outbreaks and epidemics of DENV infection. Dense human population, rapid urbanization, increasing globalization, and the tropical climatic conditions have contributed toward the frequent outbreaks of DENV infection in Delhi. Hence, a continuous serosurveillance of the circulating DENV strain is a necessity to prevent further epidemics and help in the development of local control program. $^{16}$

In the present study, molecular characterization of circulating DENV strains was performed targeting the CprM junction. Genotyping based on CprM junction is easier and economical because of the utilization of a single set of primer for both amplification and sequencing of any of the four DENV serotypes. ${ }^{9}$

The frequency of dengue observed in 2017 to 2018 in our tertiary care hospital was found to be $4.6 \%$. Though Delhi is hyperendemic for dengue, a low positivity rate (4.6\%) was found in our study. The aim of the study was to not only assess the prevalence of dengue in Delhi but also analyze the circulating serotype/genotype of DENV in the year 2017 to 2018 circulating in Delhi. So, the positivity of $4.6 \%$ is only reflective of the single-center footfall and not of entire Delhi. It is a single-center hospital-based study, therefore the positivity is lower. Moreover in the years 2017 and 2018, as there was no ongoing outbreak in the city, the overall positivity was lower. The numbers of dengue cases in 2017 and 2018 in Delhi were 9,271 and 7,136 as compared with 2015 when Delhi had experienced a dengue outbreak and the number of dengue cases reported were $15,867 .{ }^{17}$

A change in the circulation pattern of dengue serotypes has been reported from Delhi in terms of prevalent serotype. The DENV-2 serotype dominated the dengue fever during 2003 to 2006 . $^{13,18-21}$ DENV-3 emerged as a dominant serotype in the year 2003 which continued in circulation till $2006 .^{18}$ In addition, 2006 also reported the emergence of DENV-1 in $30 \%$ of the cases. ${ }^{18}$ Subsequently, the predominance of DENV-1 was reported till 2010. DENV-1 and DENV-2 serotypes cocirculated in almost equal proportions in $2011^{8}$. A switch in the prevailing serotype occurred again in 2012 due to codominant circulation of DENV-2 and DENV-3. DENV-2 dominated during 2012, 2013, and 2015.8,15 Subsequently, DENV-1 dominated in $2014^{16}$. Further, during 2016 , a change in the circulating serotype occurred leading to dominance of DENV-3 in this region ( - Table $\mathbf{1}$ ).

The most relevant finding of our study was the circulation of a single serotype, DENV-3 genotype III during 2017 to 2018 which was similar to the DENV-3 strain circulating in the year 2016. ${ }^{22}$ Similar studies from Pakistan also reported the circulation of DENV-3 during 2015. ${ }^{23}$ Studied strains of DENV-3 were clustered in genotype III (Indian subcontinent) by phylogenetic analysis. The analysis also concluded that the DENV-3 study sequences grouped with the strains from China (unpublished), Pakistan, ${ }^{24}$ and India. ${ }^{24-26}$ Furthermore DENV-3 serotype has been reported from different geographical regions including India.

The oldest DENV-3 strain, Philippines 56, was classified as genotype I along with other strains from the Philippines and 


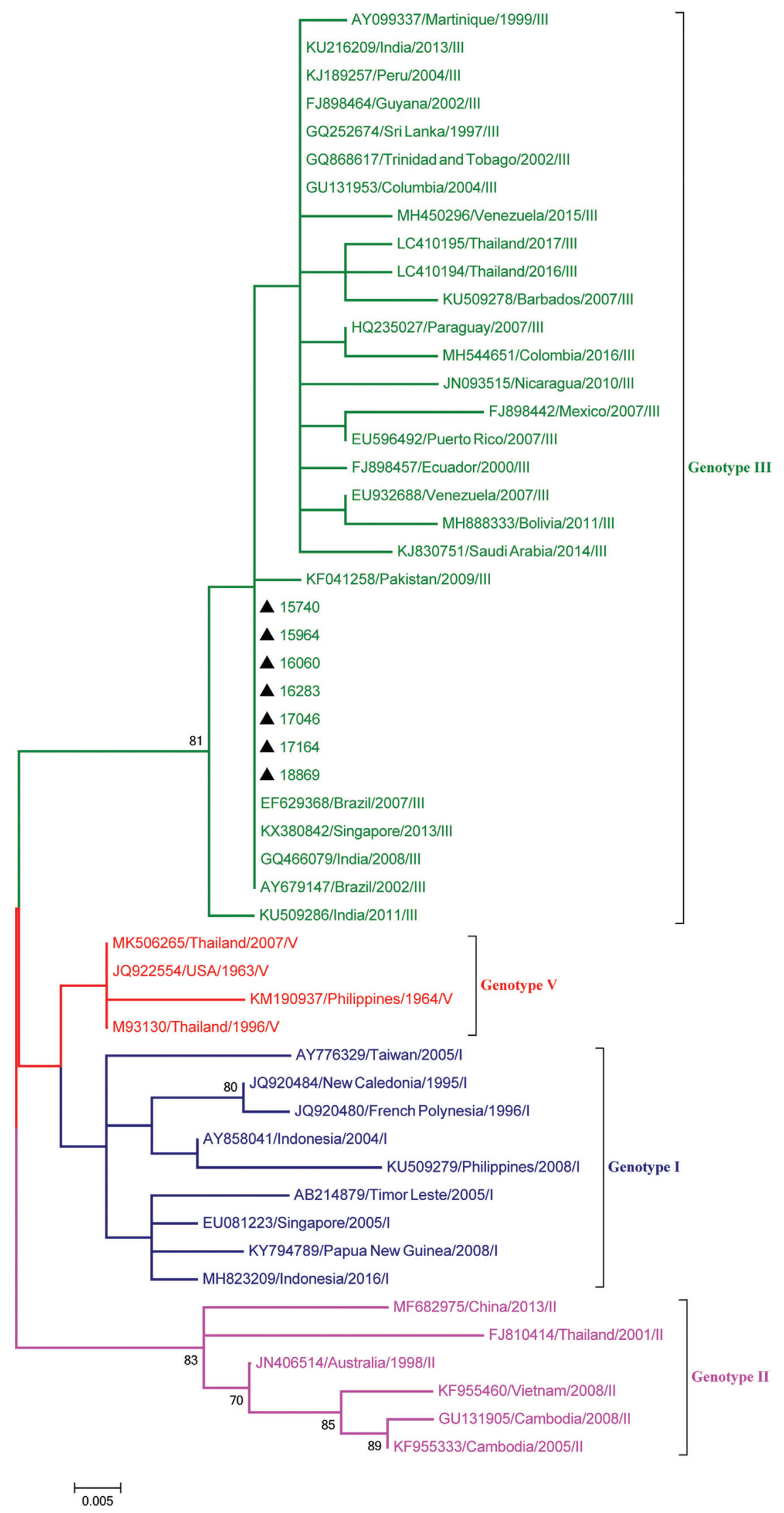

Fig. 1 Maximum likelihood phylogenetic tree of DENV-3 strains. The study sequences are marked by the symbol $\Delta$. Bootstrap values are represented by the number on nodes generated by 1,000 replications. The strains used to construct the tree are represented by the strain name, followed by country/region and year of isolation/submission of strain. The study sequences clustered with genotype III (Indian subcontinent). DENV, dengue virus. 
Table 1 Circulation of various dengue serotypes in Delhi

\begin{tabular}{|l|l|l|l|}
\hline Year & Dengue virus serotype & Epidemic/nonepidemic & References \\
\hline 1996 & 2 & Epidemic & Dar et al $^{28}$ \\
\hline 1997 & 1 & Nonepidemic & Vajpayee et al $^{29}$ \\
\hline 2003 & $1,2,3$ (genotype III),4 & Epidemic & Dar et al $^{8}$ \\
\hline 2004 & 1 & Nonepidemic & Gupta et al $^{10}$ \\
\hline 2005 & 3 (genotype III) & Epidemic & Gupta et al $^{10}$ \\
\hline 2006 & 3 (genotype III) & Epidemic & Bharaj et al $^{30}$ \\
\hline 2007 & 3 (genotype III) & Nonepidemic & Kukreti et al $^{18}$ \\
\hline 2008 & 1 & Nonepidemic & Chakravarti et al $^{31}$ \\
\hline 2010 & 1 & Nonepidemic & Gupta et al $^{11}$ \\
\hline $2011-2014$ & $1,2,3$ (genotype III) & Nonepidemic & Afreen et al $^{32}$ \\
\hline 2015 & 2 & Epidemic & Choudhary et al $^{33}$ \\
\hline 2016 & 3 (genotype III) & Nonepidemic & Parveen et al $^{22}$ \\
\hline $2017-2018$ & 3 (genotype III) & Nonepidemic & Present study $^{2}$ \\
\hline
\end{tabular}

Indonesia isolated over two decades, suggesting the persistence of this genotype in the area for a long time.

Genotype III of DENV-3 circulates throughout the world, whereas other genotypes are localized in particular geographic regions, indicating a higher potential of genotype III to spread and dominate in geographically diverse regions of the world. This genotype has also been implicated in major dengue epidemics in several parts of Asia, Africa and the Americas and has the potential to cause an international dengue pandemic. ${ }^{27}$

Furthermore our study also shows that in the nonepidemic period usually one strain circulates and cocirculation of multiple types with concurrent infection with more than one serotype is not seen.

\section{Limitations}

The study had few limitations like the study was retrospective in nature, a small sample size, and it was representative of patients coming to a tertiary care hospital. The exact depiction of data from the community is not truly represented by hospital based studies .Therefore for virological surveillance, community-based prospective studies should be performed.

\section{Conclusion}

The study provides crucial information highlighting the need for continuous monitoring of DENV genotypes in the endemic region. This study revealed circulation of DENV-3, genotype III in Delhi in the year 2017 to 2018 similar to 2008 viral type. Virological surveillance is an important exercise to be done for viral infections with public threat and outbreak potential.

Funding

None.
Conflict of Interest

None declared.

Acknowledgment

The authors would like to thank Mr. Piyush Bahuguna for his contribution in providing the data for dengue virus infection for the year 2017 to 2018 .

\section{References}

1 World Health Organization. Dengue and severe dengue. Available at: https://www.who.int/news-room/fact-sheets/detail/dengueand-severe-dengue

2 Bhatt S, Gething PW, Brady OJ, et al. The global distribution and burden of dengue. Nature 2013;496(7446):504-507

3 Guzman MG, Gubter DJ, Izquierdo A, Martinez E, Halstead SB. Dengue infection. Accessed March 14, 2020 at: https://www. nature.com/articles/nrdp201655?draft=collection\&proof=true

4 Chen R, Vasilakis N. Dengue-quo tu et quo vadis? Viruses 2011;3 (09):1562-1608

5 Gubler DJ. The global emergence/resurgence of arboviral diseases as public health problems. Arch Med Res 2002;33(04):330-342

6 Guzman MG, Vazquez S. The complexity of antibody-dependent enhancement of dengue virus infection. Viruses 2010;2(12): 2649-2662

7 Grange L, Simon-Loriere E, Sakuntabhai A, Gresh L, Paul R, Harris E. Epidemiological risk factors associated with high global frequency of inapparent dengue virus infections. Front Immunol 2014;5:280

8 Dar L, Gupta E, Narang P, Broor S. Cocirculation of dengue serotypes, Delhi, India, 2003. Emerg Infect Dis 2006;12(02): 352-353

9 Kukreti H, Mittal V, Chaudhary A, et al. Continued persistence of a single genotype of dengue virus type-3 (DENV-3) in Delhi, India since its re-emergence over the last decade. J Microbiol Immunol Infect 2010;43(01):53-61

10 Gupta E, Dar L, Kapoor G, Broor S. The changing epidemiology of dengue in Delhi, India. Virol J 2006;3:92

11 Gupta E, Mohan S, Bajpai M, Choudhary A, Singh G. Circulation of dengue virus-1 (DENV-1) serotype in Delhi, during 2010-11 after dengue virus-3 (DENV-3) predominance: a single centre hospitalbased study. J Vector Borne Dis 2012;49(02):82-85 
12 World Health Organization. Dengue Guidelines for Diagnosis, Treatment, Prevention and Control: New Edition. Geneva, Switzerland: World Health Organization; 2009

13 Lanciotti RS, Calisher CH, Gubler DJ, Chang G-J, Vorndam AV. Rapid detection and typing of dengue viruses from clinical samples by using reverse transcriptase-polymerase chain reaction. J Clin Microbiol 1992;30:7

14 Laboratory diagnosis and diagnostic tests. In: Dengue: Guidelines for Diagnosis, Treatment, Prevention and Control: New Edition. Geneva, Switzerland: World Health Organization; 2009

15 Klungthong C, Gibbons RV, Thaisomboonsuk B, et al. Dengue virus detection using whole blood for reverse transcriptase PCR and virus isolation. J Clin Microbiol 2007;45(08): 2480-2485

16 Gore MM. Need for constant monitoring of dengue infections. Indian J Med Res 2005;121(01):9-12

17 National Health Mission, India. National vector borne disease control programme. Accessed October 24, 2020 at: https:// nvbdcp.gov.in/index4.php? lang $=1$ \&level $=0$ \&linkid $=431$ \&lid $=3715$

18 Kukreti H, Chaudhary A, Rautela RS, et al. Emergence of an independent lineage of dengue virus type 1 (DENV-1) and its co-circulation with predominant DENV-3 during the 2006 dengue fever outbreak in Delhi. Int J Infect Dis 2008;12(05): 542-549

19 Dash PK, Parida MM, Saxena P, et al. Emergence and continued circulation of dengue-2 (genotype IV) virus strains in northern India. J Med Virol 2004;74(02):314-322

20 Singh P, Mittal V, Rizvi MMA, et al. The first dominant cocirculation of both dengue and chikungunya viruses during the post-monsoon period of 2010 in Delhi, India. Epidemiol Infect 2012;140(07):1337-1342

21 Singh UB, Maitra A, Broor S, Rai A, Pasha ST, Seth P. Partial nucleotide sequencing and molecular evolution of epidemic causing dengue 2 strains. J Infect Dis 1999;180(04):959-965

22 Parveen N, Islam A, Tazeen A, et al. Circulation of single serotype of dengue virus (DENV-3) in New Delhi, India during 2016: a change in the epidemiological trend. J Infect Public Health 2019; 12(01):49-56

23 Suleman M, Faryal R, Alam MM, et al. Outbreak of dengue virus type-3 in Malakand, Pakistan 2015; a laboratory perspective. Acta Trop 2017;169:202-206

24 Koo C, Nasir A, Hapuarachchi HC, et al. Evolution and heterogeneity of multiple serotypes of Dengue virus in Pakistan, 20062011. Virol J 2013;10(01):275

25 Sharma S, Dash PK, Agarwal S, Shukla J, Parida MM, Rao PVL. Comparative complete genome analysis of dengue virus type 3 circulating in India between 2003 and 2008. J Gen Virol 2011;92 (Pt 7):1595-1600

26 Dash PK, Parida MM, Saxena P, et al. Reemergence of dengue virus type-3 (subtype-III) in India: implications for increased incidence of DHF \& DSS. Virol J 2006;3:55

27 Messer WB, Gubler DJ, Harris E, Sivananthan K, de Silva AM. Emergence and global spread of a dengue serotype 3, subtype III virus. Emerg Infect Dis 2003;9(07):800-809

28 Dar L, Broor S, Sengupta S, Xess I, Seth P. The first major outbreak of dengue hemorrhagic fever in Delhi, India. Emerg Infect Dis 1999;5(04):589-590

29 Vajpayee M, Mohankumar K, Wali JP, Dar L, Seth P, Broor S. Dengue virus infection during post-epidemic period in Delhi, India. Southeast Asian J Trop Med Public Health 1999;30(03):507-510

30 Bharaj P, Chahar HS, Pandey A, et al. Concurrent infections by all four dengue virus serotypes during an outbreak of dengue in 2006 in Delhi, India. Virol J 2008;5:1

31 Chakravarti A, Kumar A, Matlani M. Displacement of dengue virus type 3 and type 2 by dengue virus type 1 in Delhi during 2008 . Indian J Med Microbiol 2010;28(04):412

32 Afreen N, Naqvi IH, Broor S, Ahmed A, Parveen S. Phylogenetic and molecular clock analysis of dengue serotype 1 and 3 from New Delhi, India. PLoS One 2015;10(11):e0141628

33 Choudhary MC, Gupta E, Sharma S, Hasnain N, Agarwala P. Genetic signatures coupled with lineage shift characterise endemic evolution of dengue virus serotype 2 during 2015 outbreak in Delhi, India. Trop Med Int Health 2017;22(07):871-880 